\title{
Electrodeposition of complex high entropy oxides via water droplet formation and conversion to crystalline alloy nanoparticles.
}

\author{
Stephen J. Percival, ${ }^{+, *}$ Ping Lu,^^ Daniel R. Lowry,, Tina M. Nenoff",* \\ + Sandia National Laboratories, Electronic, Optical and Nano Materials Department, Albuquerque, New Mexico 87185, \\ United States \\ ^ Sandia National Laboratories, Materials Characterization and Performance Department, Albuquerque, New Mexico \\ 87185, United States \\ \# Sandia National Laboratories, Materials Chemicals and Physics Center, Albuquerque, New Mexico 87185, United \\ States \\ *sperciv@sandia.gov *
}

KEYWORDS: Nanoparticles, Ligand Free, High Entropy Oxide, Electrodeposition, Emulsion Collisions, Thermal Reduction Oxygen Reduction Reaction.

\section{Supporting Information:}

The final deposition nanoparticle composition can be changed, and a desired composition targeted by adjusting the starting concentrations of the metal salts in the aqueous solution before deposition. To demonstrate this approach, we have synthesized nanoparticles with a targeted FeNiCoCrMn composition that has a much higher proportion of cobalt compared to the other metals. This was done by changing the initial proportions of the metal salts with Co having $16 \mathrm{mM}$ and the rest having $6 \mathrm{mM}$, while still maintaining a total metal salt concentration of $40 \mathrm{mM}$ (normally $8 \mathrm{mM}$ for each metal salt to achieve the equimolar composition). The resulting deposited particles are shown in Figure S1 and are seen to have a much higher cobalt content than the previously analyzed equimolar particles. As another example of this Figure S2 shows a low Cr content set of HEO NPs where the initial Cr content of the deposition solutions was much lower than the other metals.

Figure S3 shows SEM images of some of the as deposited nanoparticles on HOPG which are seen to be spherical similarly to what was observed in the STEM analysis. After annealing at $600{ }^{\circ} \mathrm{C}$ for 1 hour under inert atmosphere, the particles show a dramatic morphology change where they have become more crystalline and are no longer spherical, indicating they have been reduced to metallic nanoparticles.

After thermal annealing, the nanoparticles' structure changes and they are reduced and phase segregate into metallic FeNiCo nanoparticles with a CrMnOx phase surrounding and stabilizing them. The final size distribution of the metallic FeNiCo nanoparticles was measured from representative TEM images, as seen in Figure 4 and Figure 5 of the manuscript, and is presented in Figure S4. The histogram shows the measured nanoparticle diameters, in $\mathrm{nm}$, and that there is a positively skewed probability distribution of nanoparticle sizes with the highest population at lower sizes, which drops off at larger diameters. These measured particles represent the large majority of the particles deposited on the substrates but there are certainly some larger ones that are a few hundred nanometers as seen in the SEM images, which cannot resolve the smallest of these particles. 

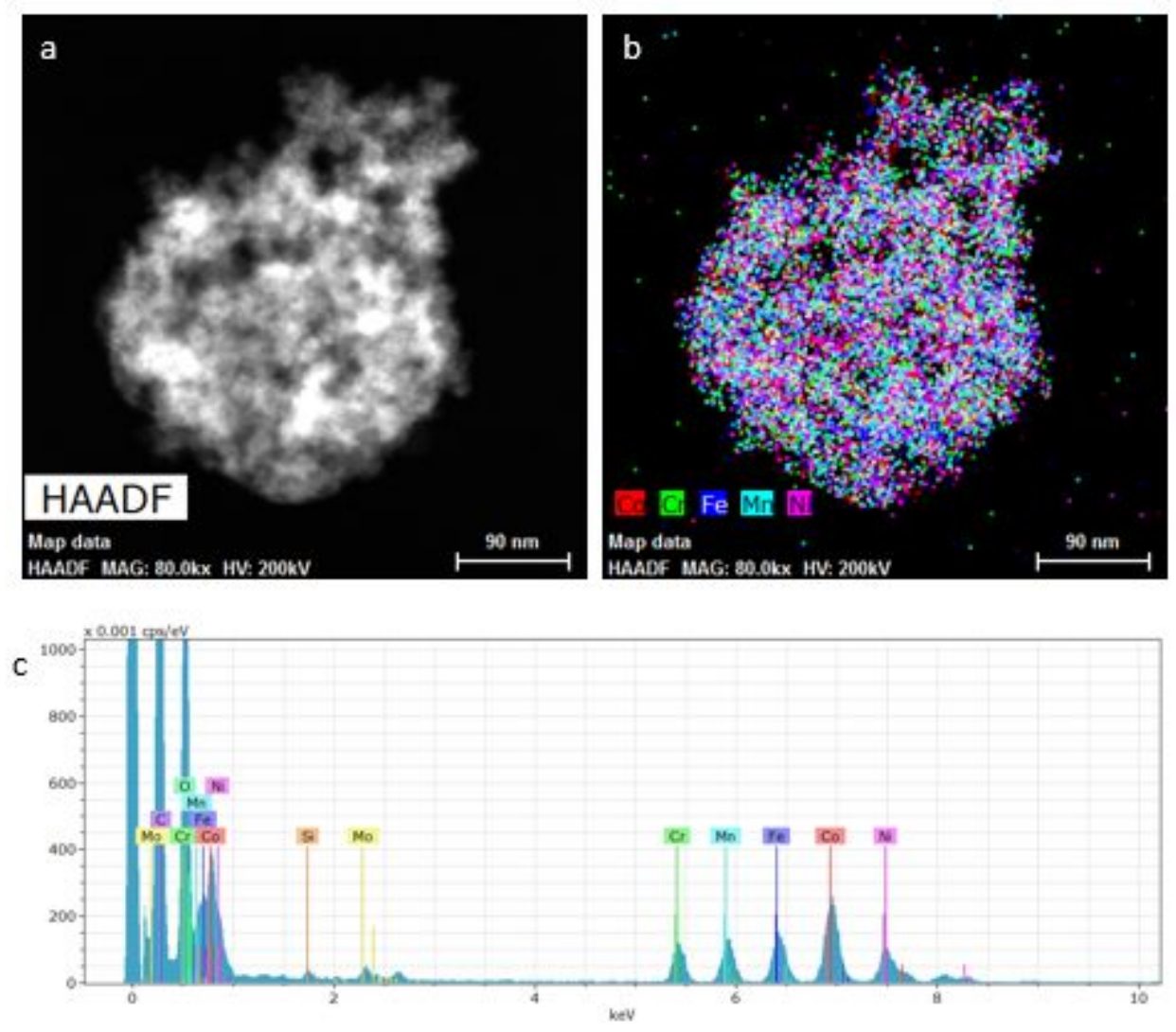

Figure S1: STEM and EDS analysis of the high Co content amorphous HEO NPs: (a) STEM HAADF image; (b) corresponding EDS color maps and (c) summed EDS spectrum from the region, showing the HEO has amorphous structure and an atomic ratio of $\mathrm{Fe}_{1.0} \mathrm{Ni}_{0.8} \mathrm{Co}_{1.5} \mathrm{Cr}_{1.0} \mathrm{Mn}_{1.0}$. Deposition applied potential was $-2.0 \mathrm{~V}$ (vs. $\mathrm{Ag} / \mathrm{AgCl}$ ). 

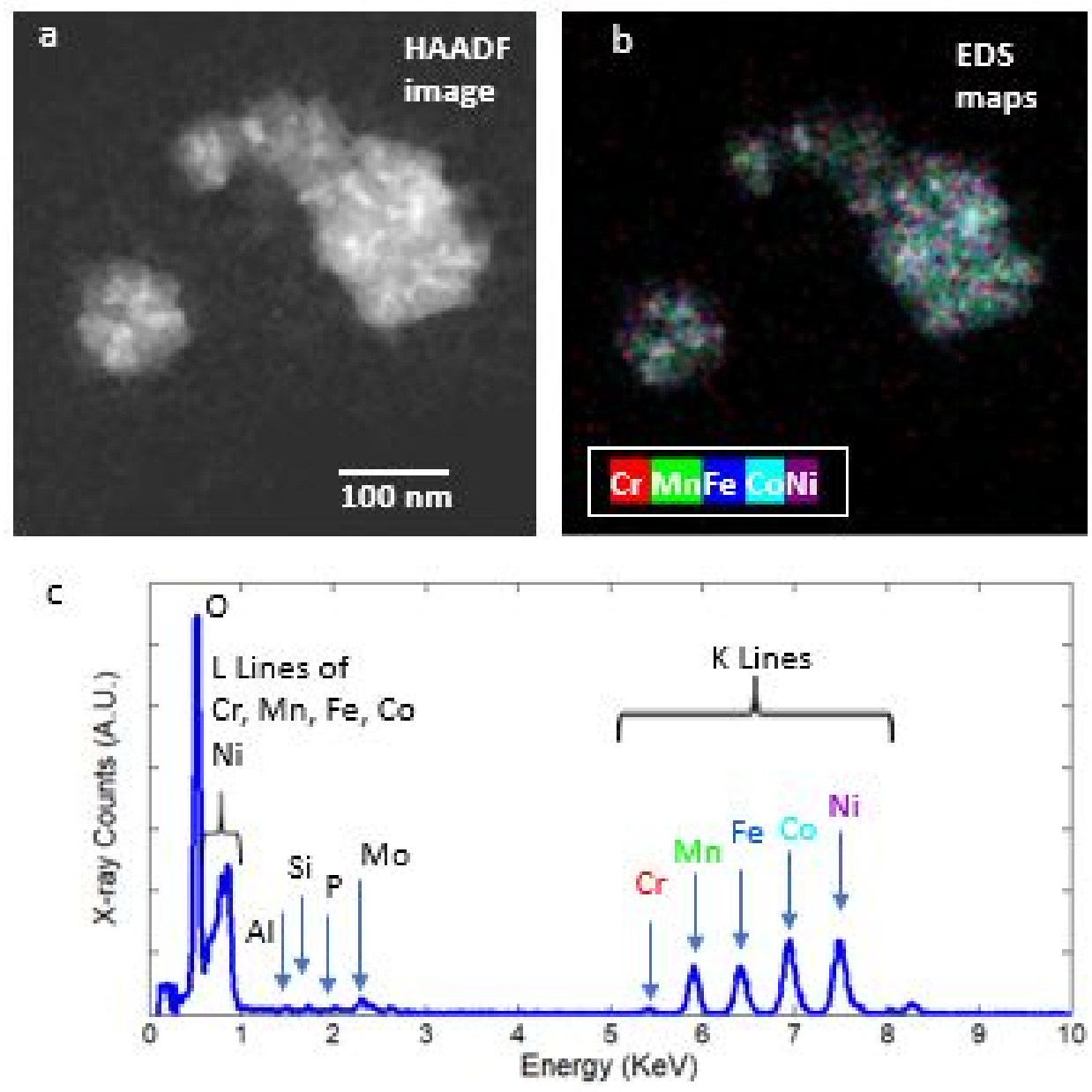

Figure S2: STEM imaging and EDS analysis of FeNiCoCrMn oxide phase. (a) HAADF image; (b) EDS composite color maps and (c) summed EDS spectrum from the particles. The HEA oxide has an estimated cation composition of $\mathrm{Fe}_{1.0} \mathrm{Ni}_{1.3} \mathrm{Co}_{1.3} \mathrm{Cr}_{0.1} \mathrm{Mn}_{1.0}$. 


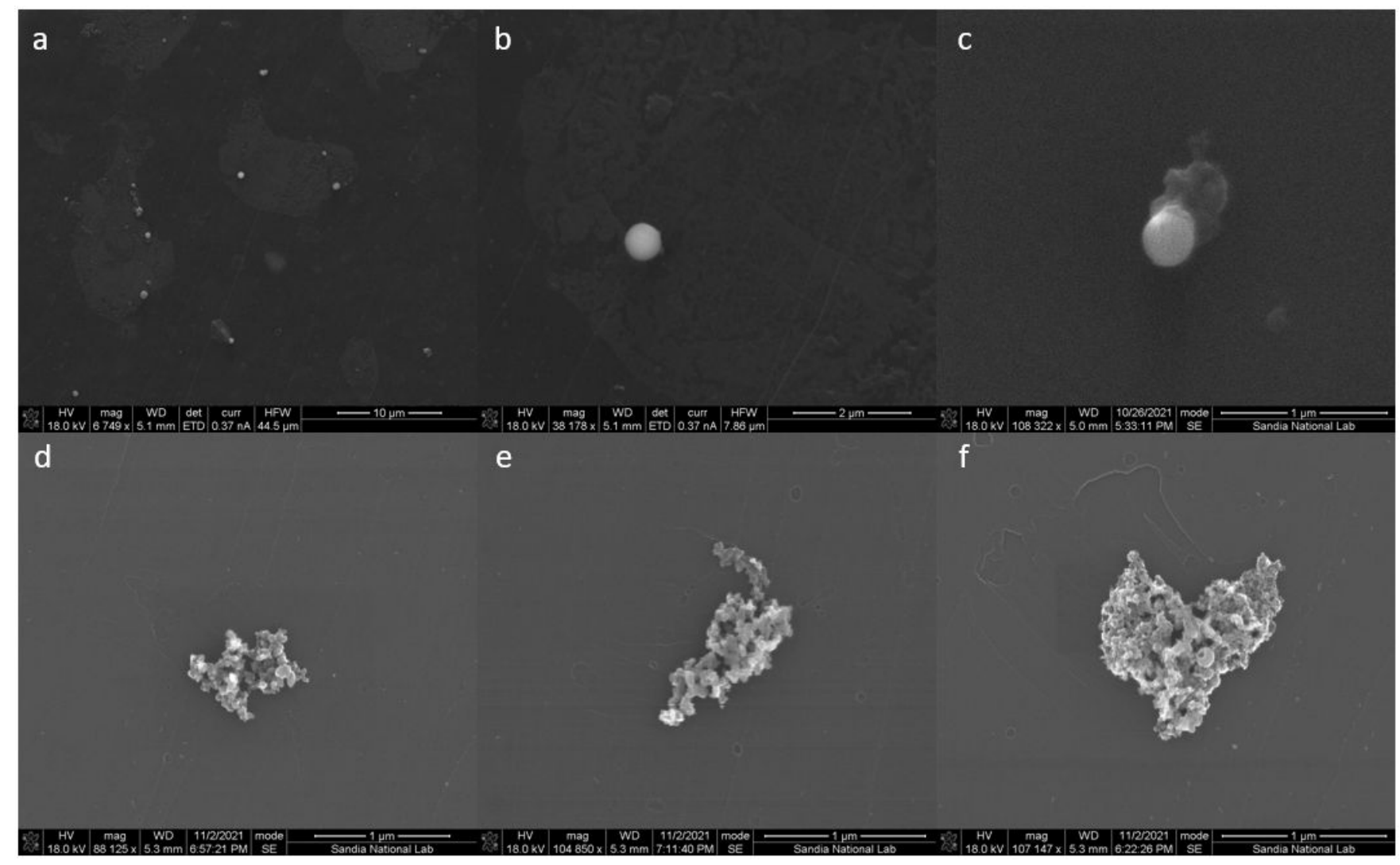

Figure S3: SEM images of the as made amorphous HEO NPs on HOPG: ( $a, b$ and $c)$ and of the annealed nanoparticles on HOPG (d, e and f). 


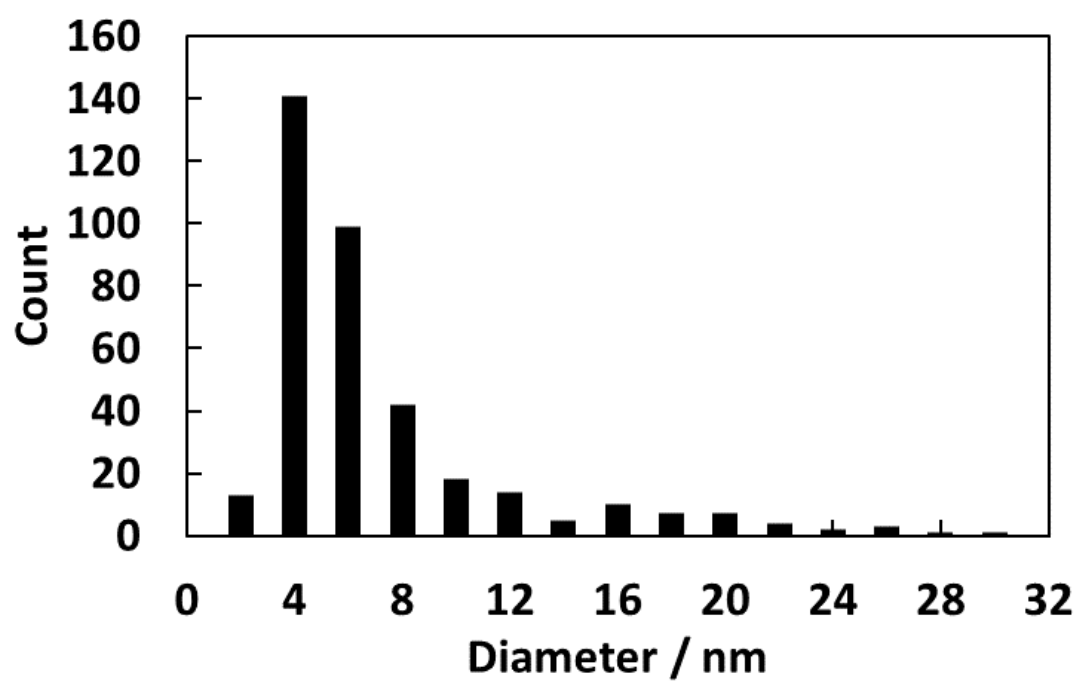

Figure S4: Histogram showing the measured size distribution of the metallic FeNiCo nanoparticles, from TEM images, after the thermal annealing step. 